\title{
PRODUÇÃO DE ÉSTERES DE ÁCIDOS GRAXOS A PARTIR DO ÓLEO DE ÇAROÇO DE ALGODÁO EM DUAS ETAPAS REACIONAIS
}

\author{
Camila da Silva \\ Caroline Portilho Trentini
}

\begin{abstract}
Resumo: Este trabalho visa estudar a produção de ésteres de ácidos graxos a partir do óleo de caroço de algodão (OCA) em duas etapas reacionais. A esterificação dos ácidos graxos livres (AGL) do óleo de caroço de algodão foi conduzida utilizando catálise ácida homogênea. Nos experimentos avaliou-se o efeito da temperatura, razão molar AGL:álcool e concentração de catalisador, sendo realizadas as reações com etanol e metanol. Os resultados obtidos demonstram maiores conversôes nas reaçóes utilizando metanol. Para ambos os alcoóis estudados as variáveis razão molar AGL:álcool e percentual de catalisador apresentam efeito positivo sobre a conversão da reação. A partir do óleo esterificado, avaliou-se a transesterificação metílica do óleo de caroço de algodáo utilizando catálise básica homogênea. Nos experimentos avaliou-se o efeito da temperatura, razáo molar óleo:metanol e concentraçáo de catalisador, considerando um planejamento experimental $3^{2}$ com triplicata do ponto central. Os melhores resultados em termos de rendimento em ésteres metílicos $(-82 \%)$ foram obtidos na temperatura de $60^{\circ} \mathrm{C}$, razão molar óleo:metanol de 1:9, $1 \%$ de catalisador e 30 minutos de reação. Com base neste resultado, a cinética reacional foi obtida reportando-se conversôes na ordem de 96\% do OCA em ésteres metílicos de ácidos graxos em 100 minutos de reação.
\end{abstract}

Palavras-chave: óleo de caroço de algodão, ácidos graxos livres, ésteres de ácidos graxos.

\begin{abstract}
This work aims to study the production of fatty acid esters from cottonseed oil (OCA) in two reaction steps. The esterification of free fatty acids (FFA) from cottonseed oil was conducted using homogeneous acid catalysis. In experiments evaluated the effect of temperature, molar ratio FFA: alcohol and catalyst concentration, the reaction being performed with ethanol and methanol. The results demonstrate higher conversions in the reactions using methanol. For both alcohols studied variables molar ratio FFA: alcohol and percentage of catalyst have positive effect on the conversion of the reaction. From the esterified oil was evaluated methyl transesterification of cottonseed oil using basic catalysis homogeneous. In experiments evaluated the effect of temperature, molar ratio of oil: methanol and concentration of catalyst, considering an experimental planning with $3^{2}$ triplicate center point. The best results in terms of yield methyl esters $(-82 \%)$ were obtained at a temperature of $60^{\circ} \mathrm{C}$, molar ratio oil: methanol 1:9, $1 \%$ catalyst and 30 minutes reaction. Based on this result, the reaction kinetics was obtained referring conversions on the order of $96 \%$ of the cottonseed oil into methyl esters of fatty acids in 100 minutes of reaction.
\end{abstract}

Keywords: cottonseed oil, free fatty acids, fatty acid esters. 


\section{INTRODUÇÃO}

$\mathrm{O}$ interesse mundial em biodiesel vem aumentando, devido o seu potencial para reduzir a dependência do diesel de petróleo, para mitigar possíveis impactos negativos da mudança climática global, diminuindo emissóes de $\mathrm{CO}_{2}$ do setor de transportes (Carraretto et al. 2004). O biodiesel (mistura de ésteres de ácidos graxos) é uma forma de energia renovável derivado de animais e recursos vegetais, obtido pela reação de transesterificação de um triglicérideo na presença de metanol ou etanol, com um catalisador adequado (Antoni et al. 2007; Durrett et al. 2008). Dentre os fatores mais comuns que influenciam a transesterificação, estão a razão molar de álcool para óleo, temperatura da reação, tipo de catalisador, tempo de reação, assim como a água e o conteúdo de ácidos graxos livres no óleo (Agarwal, 2007; Al-Zuhair, 2007; VanGerpen, 2005)

O óleo de caroço de algodão bruto apresenta elevado percentual de AGL e água tornando o processo convencional a partir da transesterificaçáo com catalisador homogêneo alcalino inviável (Vyas et al. 2010). O alto conteúdo de ácidos graxos livres ocasiona a formação de sabão, o que reduz o rendimento da reaçáo e dificulta o processo de separaçáo e purificaçáo dos produtos (Talukder et al. 2010).

Neste contexto, o objetivo deste trabalho é a produção de ésteres de ácidos graxos em duas etapas reacionais (esterificação/transesterificação) a partir do óleo provindo do caroço de algodáo por rota etílica e metílica. O efeito da temperatura, razão molar e concentraçáo de catalisador sobre a conversão da reação é reportado.

\section{MATERIAIS E MÉTODOS}

\subsection{MATERIAIS}

Neste trabalho utilizou-se como substratos o óleo de caroço de algodão (Paraguaçu Têxtil), metanol absoluto (JT Baker) e etanol absoluto (JT Baker). A composiçáo de ácidos graxos do óleo de caroço de algodáo é apresentada na Tabela 1 , sendo que o percentual de ácidos graxos livres foi de 3,23 $\%$. Como catalisadores, utilizou-se ácido sulfúrico (Quimex) na etapa de esterificação e metóxido de sódio (Evonik) na etapa de transesterificação. $\mathrm{Na}$ determinaçáo da acidez por titulação utilizou-se hidróxido de sódio (Nuclear), etanol absoluto (JT Baker), éter etílico (Vetec) e indicador fenolftaleína (Nuclear). Nas análises por cromatografia gasosa, utilizou-se heptano (F. Maia) como solvente e metil heptadecanoato (Sigma Adrich) como padrão interno.

Tabela 1: Composição em ácidos graxos do óleo de caroço de algodão.

\begin{tabular}{cc}
\hline Ácido Graxo & Percentual [\%] \\
\hline C16:0 & 22,70 \\
C16:1 & 0,18 \\
C18:0 & 2,57 \\
C18:1 & 17,74 \\
C18:2 & 56,80 \\
\hline
\end{tabular}

\subsection{PROCEDIMENTO EXPERIMENTAL}

\subsubsection{REAÇÓES DE ESTERIFICAÇÃO}

As reaçôes foram conduzidas em um balão de três bocas, onde pesou-se o óleo. Conectou-se o balão a um condensador e a uma chapa de aquecimento com agitaçáo controlada (800 rpm), e o imergiu em um banho-maria com controle de temperatura. Paralelamente pesou-se o álcool e o catalisador em um béquer. Após a temperatura de reação ser alcançada, adicionou-se o álcool/catalisador e esperou o tempo de reaçáo. Dado o tempo reacional, removeu-se o catalisador do meio reacional por lavagem com água destilada na temperatura de $80{ }^{\circ} \mathrm{C}$ até neutralização e o álcool não reagido foi evaporado em estufa com circulaçáo de ar na temperatura de $80^{\circ} \mathrm{C}$.

\subsubsection{REAÇÕES DE TRANSESTERIFICAÇÃO}

As reaçôes foram conduzidas em baláo de três bocas, onde pesou-se o óleo. Conectou-se o balão a um condensador e uma chapa de aquecimento com agitaçáo controlada $(800 \mathrm{rpm})$, e o emergiu em um banho-maria com controle de temperatura. Paralelamente pesou-se o metanol e o catalisador em um béquer. Após a temperatura de reaçáo ser alcançada, adicionou-se o metanol/ catalisador e esperou o tempo de reaçáo. Dado 
o tempo reacional adicionou-se $10 \mathrm{~mL}$ de acido sulfúrico $10 \%$ para cessar a reação, neutralizar o metóxido de sódio. Removeu-se o catalisador do meio reacional por lavagem com água destilada na temperatura de $80^{\circ} \mathrm{C}$ até neutralizaçáo e o metanol não reagido foi evaporado em estufa com circulação de ar na temperatura de $80{ }^{\circ} \mathrm{C}$.

\subsection{MÉTODOS ANALÍ́TICOS}

\subsubsection{DETERMINAÇÃO DO TEOR DE ÁCIDOS GRAXOS LIVRES}

Determinou-se o percentual de ácidos graxos livres com base ao método Ca 5a-40 (AOCS, 1998). Pesou-se $1 \mathrm{~g}$ da amostra em um erlenmeyer, adicionou-se $25 \mathrm{~mL}$ de éter/álcool e 3 gotas de indicador fenolftaleína. Titulou-se a amostra com hidróxido de sódio até a mesma apresentar coloração rósea. Anotou-se a quantidade do hidróxido de sódio utilizado na titulação (em $\mathrm{mL}$ ), calculando a o percentual de acidez conforme a equação 1 .

(1) Onde: "M" é molaridade do titulante, "V" o volume gasto para titular a amostra e o "P" gramas da amostra.

A conversão da reação foi calculada utilizando a equação (2):

(2) Onde: "Pi" é o percentual de AGL óleo, "Pf" é o percentual de AGL do óleo esterificado.

\subsubsection{DETERMINAÇÃO DO TEOR DE ÉSTERES ETÍLICOS}

O procedimento analítico foi conduzido conforme Silva et al. (2010). A amostra foi injetada em cromatógrafo a gás (Agilent GC 7890), equipado com detector de ionizaçáo de chamas e uma coluna capilar (ZB WAX, 30m x 0,25mm x 0,25mm). Utilizou-se a temperatura inicial da coluna de $120^{\circ} \mathrm{C}$, permanecendo por $2 \mathrm{~min}$ nesta condição, aumentando-se a temperatura a uma taxa de $10^{\circ} \mathrm{C} / \mathrm{min}$ até $180^{\circ} \mathrm{C}$, aumentando novamente a uma taxa de $5^{\circ} \mathrm{C} / \mathrm{min}$ até $210^{\circ} \mathrm{C}$ permanecendo 1 min nesta condição e finalmente aumentando-se a uma taxa de $15^{\circ} \mathrm{C} / \mathrm{min}$ até $230^{\circ} \mathrm{C}$ permanecendo nesta condiçãao por $2 \mathrm{~min}$. O Hélio foi utilizado como gás de arraste, a temperatura do injetor e detector de $250^{\circ} \mathrm{C}$ e a taxa de split de 1:50.

\section{RESULTADOS E DISCUSSÓES}

\subsection{REAÇÃO DE ESTERIFICAÇÃO}

Os resultados em relação ao efeito das variáveis de processo (temperatura, razão molar AGL:álcool e percentual de catalisador) sobre a conversáo da reação de esterificação são apresentados na Tabela 2, bem como as condiçóes experimentais estudadas. A partir dos resultados, verificam-se maiores conversóes na utilização de metanol como substrato da reação, sendo que efeito similar foi obtido por Farag et al. (2011), Pisarello et al. (2010), Canakci e Van Gerpen (2001). O metanol é mais amplamente empregado na produçáo de biodiesel, por ser mais reativo, implica em menor temperatura e tempo de reação (Lôbo et al. 2009).

Tabela 2: Condiçóes experimentais e resultados das reaçôes de esterificação.

\begin{tabular}{cccccc}
\hline Exp & Álcool & $\mathbf{T}[\mathbf{o} \mathbf{C}]$ & $\mathbf{R}$ & $\mathbf{C}[\%]$ & $\mathbf{X}[\%]$ \\
\hline 1 & Metanol & 40 & $1: 3$ & 1,5 & 28,95 \\
2 & Metanol & 40 & $1: 9$ & 1,5 & 88,19 \\
3 & Metanol & 60 & $1: 9$ & 1,5 & 88,14 \\
4 & Metanol & 60 & $1: 9$ & 0,5 & 82,26 \\
5 & Etanol & 40 & $1: 3$ & 1,5 & 13,58 \\
6 & Etanol & 40 & $1: 9$ & 1,5 & 39,85 \\
7 & Etanol & 60 & $1: 9$ & 1,5 & 44,17 \\
8 & Etanol & 60 & $1: 9$ & 0,5 & 33,49 \\
\hline
\end{tabular}

Onde: $\mathrm{T}$ - temperatura, $\mathrm{R}$ - razão molar AGL:álcool; C concentraçâo de catalisador; $\mathrm{X}$ - conversão dos ácidos graxos livres.

Farag et al. (2011) obtiveram conversóes de $60 \%$ e $90 \%$ respectivamente para o etanol e metanol utilizando ácidos graxos de soja e girassol na proporção de $1: 1$ a $60^{\circ} \mathrm{C}$ razáo molar álcool/óleo $6: 1,2.5 \%$ de ácido sulfúrico como catalisador, em 60 minutos de reação, e agitação de $300 \mathrm{rpm}$. Pisarello et al. (2010) trabalhou com ácidos graxos de óleo de girassol obtendo $-85 \%$ e $-94 \%$ de rendimento, respectivamente para o etanol e metanol, na temperatura de $60^{\circ} \mathrm{C}$, razão molar álcool:óleo 15:1, 0,2\% de ácido sulfúrico como catalisador, e 60 minutos de reação com uma agitação de 400 rpm. Canakci e Van Gerpen (2001) utilizou gor- 
dura animal com $20 \%$ de ácido palmítico, obtendo conversôes de aproximadamente $92 \%$ e $95 \%$ para o ácido palmítico respectivamente para o etanol e metanol, sendo a temperatura da reaçáo com metanol de $60^{\circ} \mathrm{C}$ e com etanol a $75^{\circ} \mathrm{C}$, razão molar álcool:óleo de 9:1, 5\% de acido sulfúrico como catalisador e 60 minutos de reação.

Entre as variáveis estudadas, verifica-se o efeito pronunciado da razão molar AGL:álcool e percentual de catalisador. Em relação à razão molar, este efeito é observado nos experimentos 1 e 2 nas reaçóes com metanol e nos experimentos 5 e 6 utilizando etanol. O aumento da proporção de catalisador no meio reacional favorece o alcance de maiores rendimentos para a reação de esterificação. O efeito positivo do percentual de ácido sulfúrico como catalisador em reaçóes de esterificação pode ser verificado nos trabalhos de Berrios et al. (2010), Farag et al. (2011) e Charoenchaitrakool e Thienmethangkoon (2011).

Berrios et al. (2010) obtiveram aproximadamente $84 \%$ de conversão dos AGL do óleo de girassol, na temperatura de $60^{\circ} \mathrm{C}$, razão molar de óleo:metanol de 1:80, 5\% de ácido sulfúrico como catalisador, em 60 minutos de reação. Thuruvengadaravi et al. (2009) relataram conversão de $85 \%$ dos AGL do óleo de semente de Pangomia pennata, na temperatura de $60^{\circ} \mathrm{C}$, razão molar óleo:metanol de 1:9, 1\% de ácido sulfúrico como catalisador, em 60 minutos de reação. Veljkovic et al. (2006) reportam aproximadamente $94 \%$ de conversão dos AGL óleo de semente de tabaco, na temperatura de $60^{\circ} \mathrm{C}$ razão molar óleo:metanol $1: 13,2 \%$ de ácido sulfúrico como catalisador, em 50 minutos de reação.

Para etapa de transesterificação utilizou-se o óleo caroço de algodão esterificado nas seguintes condições reacionais: temperatura de $60^{\circ} \mathrm{C}$, razão molar óleo:metanol 1:9 e 1,5 \% ácido sulfúrico como catalisador, o que garantiu que o teor de AGL fosse $<0,5 \%$.

\subsection{REAÇÓES DE TRANSESTERIFICAÇÃO}

As condições experimentais estudadas e os resultados obtidos em relação à reação de transesterificação do OCA esterificado estão apresentados na Tabela 3, a partir destes dados, determinou-se a influência das variáveis de processo sobre a conversão em ésteres, cujos dados são apresentados na
Tabela 4, mantendo-se fixo o tempo de contato em 30 minutos.

Tabela 3: Condições experimentais e resultados das reaçóes de transesterificação do OCA esterificado.

\begin{tabular}{ccccc}
\hline Exp & $\mathbf{T ~}\left[\mathbf{o}^{\mathbf{C}}\right]$ & $\mathbf{C}[\%]$ & $\mathbf{R}$ & $\mathbf{X}[\%]$ \\
\hline 1 & 50 & 0,5 & $1: 6$ & 65,27 \\
2 & 70 & 0,5 & $1: 6$ & 65,21 \\
3 & 50 & 1,5 & $1: 6$ & 78,75 \\
4 & 70 & 1,5 & $1: 6$ & 77,99 \\
5 & 50 & 0,5 & $1: 12$ & 58,99 \\
6 & 70 & 0,5 & $1: 12$ & 76,11 \\
7 & 50 & 1,5 & $1: 12$ & 75,00 \\
8 & 70 & 1,5 & $1: 12$ & 81,32 \\
$9(\mathrm{C})$ & 60 & 1,0 & $1: 9$ & 81,59 \\
$10(\mathrm{C})$ & 60 & 1,0 & $1: 9$ & 81,60 \\
$11(\mathrm{C})$ & 60 & 1,0 & $1: 9$ & 81,03 \\
\hline
\end{tabular}

Onde: $\mathrm{T}$ - temperatura, $\mathrm{R}$ - razão molar AGL:álcool; C concentração de catalisador; X - conversão em esteres metílicos.

Tabela 4: Análise dos Efeitos das variáveis independentes para as reações de transesterificação do OCA esterificado.

\begin{tabular}{ccc}
\hline Variável & Efeito & $\mathbf{P}$ \\
\hline Média & 74,81 & 0,000002 \\
T & 5,65 & 0,001669 \\
R & 11,87 & 0,000379 \\
C & 1,05 & 0,044984 \\
T-R & $-2,87$ & 0,006410 \\
T-C & 6,07 & 0,001451 \\
R-C & $-1,26$ & 0,032077 \\
\hline
\end{tabular}

A partir da análise dos efeitos apresentada na Tabela 4 observa-se que as variáveis estudadas apresentaram efeito significativo e positivo sobre a conversão da reação $(\mathrm{p}<0,05)$ sendo que a razão molar e a temperatura apresentaram efeito mais pronunciado. Com base nos resultados apresentados na Tabela 3, rendimento de aproximadamente $82 \%$ é obtido nas condiçôes do ponto central. Observando-se efeito significativo da razão molar e na temperatura similar nos trabalhos de Refaat et al. (2008), Rashid et al. (2009), Cheng et al. (2004), Uzun et al. (2012) e Leung e Guo (2006).

Refaat et al. (2008) obtiveram conversóes de aproximadamente $72 \%$ e $91 \%$, a $25^{\circ} \mathrm{C}$ e $65^{\circ} \mathrm{C}$ respectivamente com o óleo de girassol, sendo a razão 
molar de óleo:metanol 1:6, 1\% hidróxido de sódio como catalisador em 60 minutos de reaçáo. A razão molar óleo:metanol 1:6 e 1:9 proporcionaram conversóes de aproximadamente $91 \%$ e $58 \%$ nas mesma condiçôes reacionais. Rashid et al. (2009) reportam uma conversáo de aproximadamente $88 \%$ e $95 \%$, a $50^{\circ} \mathrm{C}$ e $65^{\circ} \mathrm{C}$ respectivamente, utilizando o óleo do caroço de algodão, razão molar metanol:óleo 1:6, 0,75\% de metóxido de sódio como catalisador, em 60 minutos de reaçáo. A razão molar óleo:metanol 1:3 e 1:6 resultaram em respectivamente $58 \%$ e $95 \%$ em éteres, nas mesmas condiçóes reacionais. Cheng et al. (2004) reportam conversóes de aproximadamente $76 \%$ e $98 \%$ com o óleo de palma, nas temperaturas de $50^{\circ} \mathrm{C}$ e $60^{\circ} \mathrm{C}$ respectivamente, razão molar óleo:metanol de 1:10, $3 \%$ hidróxido de sódio como catalisador, 60 minutos de reação. A razão molar óleo:metanol 1:6 e 1:10 rendeu respectivamente $90 \%$ e $98 \%$, nas mesmas condiçóes reacionais. Uzun et al. (2012) obtiveram conversóes de aproximadamente $89 \%$ e $96 \%$, a $25^{\circ} \mathrm{C}$ e $50^{\circ} \mathrm{C}$ respectivamente, razão molar óleo:metanol 1:7,5, 0,5\% de hidróxido de só- óleo:metanol 1:7, 1\% de hidróxido de sódio como catalisador, em 15 minutos de reação. A razão molar óleo:metanol 1:3 e 1:7 proporcionou conversóes de $96 \%$ e $80 \%$, a $70^{\circ} \mathrm{C}, 1 \%$ de hidróxido de sódio como catalisador em 15 minutos de reação.

Com base nos resultados obtidos (Tabela 3) a cinética reacional foi obtida na condição de ponto central, cujos resultados são apresentados Figura 1. Os resultados apresentados na Figura 1 reportam rendimentos em ésteres de aproximadamente $96 \%$ para tempos de reação acima de 100 minutos.

Vicente et al. (2004) reportam $90 \%$ de conversão na transesterificação de óleo de girassol a $65^{\circ} \mathrm{C}$, razão molar óleo:metanol de 1:9, $1 \%$ de metóxido de sódio e 20 minutos de reação. Charoenchaitrakool e Thienmethangkoon (2011) na utilizaçáo de $\mathrm{KOH}$ como catalisador na metanólise de óleo de fritura obtiveram $82 \%$ de conversão em 60 ${ }^{\circ} \mathrm{C}$, razão molar de 1:9, 1,5\% de catalisador e 30 minutos de reação. Uzun et al. (2012) obtiveram aproximadamente $90 \%$ de conversão na transesterificaçáo do óleo de fritura com metanol a $60^{\circ} \mathrm{C}$,

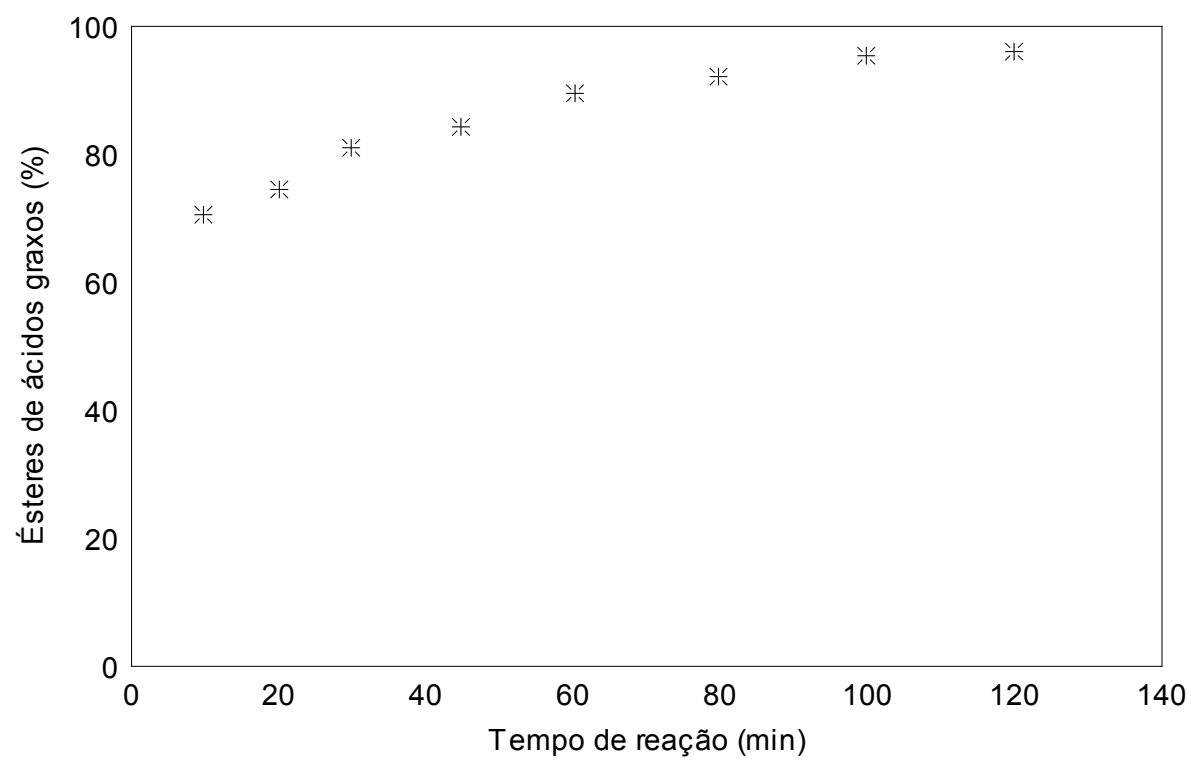

Figura 1: Cinética da reação de transesterificação do OCA após etapa de esterificação na temperatura de $60^{\circ} \mathrm{C}$, razão molar óleo metanol de 1:9 e percentual de catalisador de 1,0\%.

dio como catalisador, em 30 minutos de reação. A razão molar óleo:metanol 1:3 e 1:7,5 rendeu conversóes de $53 \%$ e $94 \%$ respectivamente, a $70^{\circ} \mathrm{C}$, $0,5 \%$ de hidróxido de sódio como catalisador, em 30 minutos de reação. Leung e Guo (2006) reportam conversôes de 805 e $96 \%$ com o óleo de canola, a $50^{\circ} \mathrm{C}$ e $70^{\circ} \mathrm{C}$ respectivamente, razão molar razão molar 1:9, 0,5\% de hidróxido de sódio como catalisador em 30 minutos de reação. Nakpong et al. (2010) obtiveram conversão de aproximadamente $94 \%$ com o óleo de pinhão manso, a $60^{\circ} \mathrm{C}$ em uma razão molar de óleo:metanol de 1:6, utilizando hidróxido de sódio a 40

1,0\% como catalisador, em 30 minutos de 
reação. Nabi et al. (2009) obtiveram conversão de $77 \%$ utilizando o óleo do caroço de algodão, a $60^{\circ} \mathrm{C}$ em uma razão molar óleo:metanol de 1:20, $0,5 \%$ de hidróxido de sódio como catalisador em 8 horas de reação. Georgogianni et al. (2007) reportam conversão de $95 \%$ da mistura do óleo de fritura de soja e algodáo na proporção de $1: 1$, a $60^{\circ} \mathrm{C}$, razáo molar óleo:metanol 1:7, 2\% de hidróxido de sódio como catalisador, em 60 minutos de reaçáo e agitação de $600 \mathrm{rpm}$. Mothé et al. (2005) obtiveram conversão de aproximadamente $94 \%$ na transesterificação do óleo de mamona com etanol, a $50^{\circ} \mathrm{C}$, razão molar óleo:etanol de 1:5, utilizando $5 \%$ de hidróxido de sódio como catalisador e 60 minutos de reação. Alamu et al. (2008) apresentam conversóes de $96 \%$ com o óleo de palma, a $60^{\circ} \mathrm{C}$, razão molar óleo:etanol 1:5, 1,0\% de hidróxido de potássio como catalisador e 120 minutos de tempo de reação. Mendow et al. (2011) reportam uma conversão de aproximadamente $98 \%$ do óleo de girassol e sebo bovino, a $55^{\circ} \mathrm{C}$, razáo molar de óleo:etanol de 1:4, 1,0\% de metóxido de sódio como catalisador e 60 minutos de reação.

\section{CONCLUSÓES}

A produção de ésteres de ácidos graxos a partir do óleo de caroço de algodão em duas etapas reacionais é reportada. Nas reaçôes de esterificação de ácidos graxos livres do óleo de caroço de algodão utilizando catálise ácida homogênea, verificou-se melhores resultados na utilizaçáo de metanol e que as variáveis razão molar AGL:álcool e concentração de catalisador apresentam efeito positivo e significativo sobre a conversão dos AGL. As maiores conversôes foram obtidas utilizando a rota metílica nas seguintes condiçôes: razão molar óleo:metanol 1:9, 1,5\% de ácido sulfúrico com catalisador, temperatura de $60^{\circ} \mathrm{C}$ e $40^{\circ} \mathrm{C}$, ambas resultaram em $88 \%$ de conversão dos AGL.

A transesterificação do óleo caroço de algodão após etapa de esterificação, utilizando metóxido de sódio como catalisador foi investigada. Verificou-se o efeito significativo das variáveis estudadas, observando o efeito significativo e positivo da razão molar e da temperatura sobre a conversão, obtendo rendimento em ésteres metílicos de -82 $\%$. No estudo da cinética da reação da conversão dos ésteres de ácidos graxos aumentou com o tempo sendo $96 \%$ de conversão dos triglicerídeos em ésteres metílicos em 100 minutos de reação.

\section{REFERÊNCIAS}

AOCS - American Oil Chemists Society. Official methods and recommended practices of the AOCS. Champaign: A.O.C.S., 1998.

AGARWAL, A.K. Biofuels (alcohols and biodiesel) applications as fuels for internal combustion engines. Progress in Energy and Combustion Science, v. 33, p.233-271, 2007.

ALAMU, O.J.; WAHEED, M. A.; JEKAYINFA, S. O. Effect of ethanol-palm kernel oil ratio on alkali-catalyzed biodiesel yield. Fuel, v. 87, p.1529-1533, 2008.

ANTONI, D.; ZVERLOV, V. V.; $\underline{\text { SCHWARZ, }}$ W.H. Biofuels from microbes. Applied Microbiology and Biotechnology, v.77, n.1, p.23-35, 2007.

AL-ZUHAIR, S. Production of biodiesel: possibilities and challenges. Biofuels, Bioproducts and Biorefining, v. 1, n. 1, p. 57-66, 2007.

ARANDA, D. A. G.; SANTOS, R. T. P.; TAPANES, N. C. O.; RAMOS, A. L. D.; ANTUNES, O. A. C. Acid-Catalyzed Homogeneous Esterification Reaction for Biodiesel Production from Palm Fatty Acids. Springer, v. 122, p.20-25, 2008.

BERRIOS, M.; MARTIN, M.A.; CHICA, A.F; MARTIN, A. Study of esterification and transesterification in biodiesel production from used frying oils in a closed system. Chemical Engineering Journal, v.160, p.473-479, 2010.

CANAKCI, M.; VAN GERPEN, J. Biodiesel Production from Oils and Fats with High Free Fatty Acids. American Society of Agricultural Engineers, v. 44, p.1429-1436, 2001.

CARRARETTO, C.; MACOR, A.; MIRANDOLA, A.; STOPPATO, A.; TONON, S. Biodiesel as alternative fuel: experimental analysis and energetic evaluations. Energy, v. 29, p.21952211,2004 .

CHAROENCHAITRAKOOL, M.; THIENMETHANGKOON, J. Statistical optimization for biodiesel production from waste frying oil 
through two-step catalyzed process. Fuel Processing Tecnhology, v.92, p. 112-118, 2011.

CHENG, S. F.; CHOO Y. M.; MA A. N.; CHUAH C. H. Kinetics study on transesterification of palm oil. Journal of Oil Palm Research, v. 16, n. 2, p.19-29, 2004.

DURRETT, T. P.; BENNING C.; OHLROGGE I. Plant triacylglycerols as feedstocks for the production of biofuels. Plant Journal, v.54, n. 4 , p.593-607, 2008.

FARAG, H. A.; EL-MAGHRABY, A.; TAHA, N. A. Optimization of factors affecting esterification of mixed oil with high percentage of free fatty acid. Fuel Processing Tecnhology, v.92, p.507-511, 2011.

GEORGOGIANNI, K. G.; KONTOMINAS, M. G.; TEGOU, E.; AVLONITIS, D.; GERGIS, V. Biodiesel Production: Reaction and Process Parameters of Alkali-Catalyzed Transesterification of Waste Frying Oils. Energy \& Fuels, v.21, p. 30233027, 2007.

LEUNG, D. Y. C.; GUO, Y. Transesterification of neat and used frying oil: Optimization for biodiesel production. Fuel Processing Technology, v. 87, p. 883-890, 2006.

LÔBO, I. P.; FERREIRA, S. L. C.; CRUZ, R. S. Biodiesel: Parâmetros de Qualidade e Métodos Analíticos. Química Nova, v. 32, n. 6, p.15961608, 2009.

LU, H.; LIU, Y.; ZHOU, H.; YANG, Y.; CHEN, M.; LIANG, B. Production of biodiesel from Jatropha curcas L. oil. Computers and Chemical Engineering, v. 33, p. 1091-1096, 2009.

MENDOW, G.; VEIZAGA, N. S.; SANCHEZ, B. S.; QUERINI, C. A. Biodiesel production by two-stage transesterification with ethanol. Bioresource Technology, v. 102, p.10407-10413, 2011.

MOTHÉ, C. G.; CORREIA, D. Z.; CASTRO, B. C. S.; CAITANO, M. Otimização da produçáo de biodiesel a partir de óleo de mamona. Revista Analytica, n. 19, p.40-44, 2005.
NABI, M. N.; RAHMAN, M. M.; AKHTER, M. S. Biodiesel from cotton seed oil and its effect on engine performance and exhaust emissions. Applied Thermal Engineering, v.29, p.2265-2270, 2009.

NAKPONG, P.; WOOTTHIKANOKKHAN, $S$. Optimization of biodiesel production from $\mathrm{Ja}$ tropha curcas L. oil via alkali-catalyzed methanolysis. Journal of Sustainable Energy \& Environment, v. 1, p.105-109, 2010.

PISARELLO, M.L.; COSTA,B. D.; MENDOW, G.; QUERINI, C. A. Esterification with ethanol to produce biodiesel from high acidity raw materials: Kinetic studies and analysis of secondary reactions. Fuel Processing Technology, v.91, p.10051014, 2010.

RASHID, U.; ANWAR, F.; KNOTHE, G. Evaluation of biodiesel obtained from cottonseed oil. Fuel Processing Technology, v. 90, p.1157-1163, 2009.

REFAAT, A. A.; ATTIA, N. K.; SIBAK, H. A.; EL SHELTAWY, S. T.; EL DIWANI, G. I. Production optimization and quality assessment of biodiesel fromwaste vegetable oil. International Journal Environmental Science and Technology, v. 5, n.1, p.75-82, 2008.

TALUKDER, M. R.; WU, J. C.; CHUA, L. P. L. Conversion of Waste Cooking Oil to Biodiesel via Enzymatic Hydrolysis Followed by Chemical Esterification. Institute of Chemical and Engineering Sciences. Energy Fuels, v.24, p.2016-2019. 2010.

THIRUVENGADARAVI, K. V.; NANDAGOPAL, J.; BALA, V. S. S.; KIRUPHA, S. D.; VIJAYALAKSHMI, P.; SIVANESAN, S. Kinetic study of the esterification of free fatty acids in non-edible Pongamia pinnata oil using acid catalyst. Indian Journal of Science and Technology, v. 2, n. 12, p.20-24, 2009.

UZUN, B. B.; KILIÇ, M.; OZBAY, N.; PUTUN, A. E.; PUTUN, E. Biodiesel production from waste frying oils: Optimization of reaction parameters and determination of fuel properties. Energy, v.44, p.347-351, 2012. 
VAN GERPEN, J. Biodiesel processing and production. Fuel Processing Technology, v. 86, p.1097-1107, 2005.

VELJKOVIC, V.B.; LAKICEVIC, S.H.; STAMENKOVIC, O.S.; TODOROVIC, Z.B.; LAZIC, M.L. Biodiesel production from tobacco (Nicotiana tabacum L.) seed oil with a high content of free fatty acids. Fuel, v. 85, p. 2671-2675, 2006.
VICENTE, G.; MARTÍNEZ, M.; ARACIL, J. Integrated biodiesel production: a comparison of different homogeneous catalysts systems. Bioresource Tecnhnology, v. 92, p. 297-305, 2004.

VYAS A. P.; VERMA J.L.; SUBRAHMANYAM N. A review on FAME production processes. Fuel, v. 89, p. 1-9, 2010. 